\title{
SENILE CATARACT - A STUDY OF THE BIOLOGY AND CHEMISTRY OF THE CRYSTALLINE LENS*
}

BY

Daniel B. Kirby, M.D., and Renee v. E-Wiener, Ph.D.

MANY diverse methods of approaching the complex subject of senile cataract have been used. Research into the control of the transparency of the crystalline lens and the development of opacities requires a working hypothesis. This paper deals with a joint consideration of the biology and chemistry of the crystalline lens, both in the normal and abnormal states and with an hypothesis of the development of cataract.

\section{Outline}

1. Growth and structural changes in the normally ageing lens.

2. Chemistry and nutrition of the normal lens.

3. Analogous changes observed in other cells and organs, especially cells of bradytrophic tissues. Changes in permeability in ageing cells. Rôle of calcium and lipoids in permeability changes. Hereditary factors influencing the ageing of an organ or part of an organ.

4. Discussion of cataract with formation of hypothesis of development of cataract.

5. Conclusions.

\section{1.-Growth and Structural Changes in the Normally Ageing Lens}

The origin and development of the crystalline lens. - The lens is of ectodermal origin. The human "Anlage," a thickening of the ectoderm after contact with the optic vesicle, is visible at the 15 th to 18th day of embryonic life. The posterior cells of the lens vesicle differentiate into the primitive cortex. Further growth and formation of the lens fibres is accomplished by the cells of the vortex at the equatorial region. The anterior epithelial cells

\footnotetext{
- The material for this thesis was collected while Daniel B. Kirby was Research Fellow in Ophthalmology for the American Academy of Ophthalmology and OtoLaryngology, and $R$. von E-Weiner was Consultant Research Chemist to the Department of Ophthalmology, Columbia University.

From the Department of Ophthalmology, College of Physicians and Surgeons, Columbia University, New York City, New York.
} 
probably divide, and move out radially to furnish cells for the vortex. It is here that the lens fibres are continually developed.

The crystalline lens capsule.-The embryonic cuticula is formed as a secretion or deposit by the lens epithelial cells and is developed into the lens capsule. The latter is a strong, glass-like, semipermeable membrane, varying in thickness, being thinnest at the posterior pole. The adult capsule is formed by accretion from the inside layers of similar material produced by the lens epithelial cells. It is undoubtedly first in semi-liquid form and is forced under capillary pressure around and between the cortex and the previously existing capsule, fusing with the latter. This mechanism of formation explains the thinness of the posterior as compared with the anterior capsule. The degree and selectivity of permeability of the capsule are important factors in the health of the lens. The lens epithelium, which continues to form new cells throughout life, is present only under the anterior part of the capsule, and extends somewhat beyond the equator.

The crystalline lens epithelium and new lens fibres.-The anterior and vortex epithelial cells represent the only nucleated, reproducing elements in the lens. The young lens fibres, which lie at the periphery of the lens, have smooth edges, are rounded or ovoid on cross section and contain a nucleus. The nuclei of these fibres degenerate gradually, as their fibres are pushed toward the interior by the newly-formed fibres. The nuclei are first fragmented into small, intensely staining particles and then gradually disappear.

The crystalline lens cortex.-The crystalline lens cortex represents the softer, more recently-formed lamellae of lens fibres, the most recent being outermost. The younger fibres contain more water and the interfibrillar diffusion spaces are more open than those of the nucleus. The relative amount of soft cortex and hard nuclear material varies with the age and the individual tendency to sclerosis of the nucleus, either physiological or pathological. The fibres are six-cornered, flattened cells, and the older fibres have finely denticulated edges. The fibres may be pictured as flat tubules, which in the cortical portion of the lens are about 10 to 12 micra wide, 2 to 3 micra thick, while their length may be measured in millimetres. The interior of the fibres consists of a clear, rather viscous colloid substance, the lens protein. The lens fibres may be likened to red blood corpuscles in their structure, because of the absence of nuclei in the mature fibres and the apparent lipoid membrane. Between the fibres there is a cement substance which stains in the familiar way with silver nitrate.

The crystalline lens nucleus. - The crystalline lens nucleus represents the more dehydrated, hardened, older portion of the lens. The lens fibres, which in the advancing period of life have been 
sub-capsular, are later pressed to the central zone. Formations, typical of various stages of development, may often be distinguished by their accretion boundaries, zones and sutures. The hardened nuclear formations develop irregularities of interfaces, pressure surfaces, colorations and refraction-index differences which may produce nuclear haze of beginning cataract.

Transparency of tissue.-The transmission of light through a perfectly transparent tissue is effected without scattering. This perfect transmission rests upon the structural organization of its constituent molecules, as well as the aggregation and direction of its micelles and cells. In the early embryonic state, the lens represents a sol and the dispersed phase with exhibition of Brownian movement of the micelles may be demonstrated with the aid of the ultramicroscope. In the later embryonic state the transformation of the sol to a gel takes place. In this process there may be a partial reorientation of the constituent micelles. The hydration of the proteins increases and in the mature lens most of the dispersion medium is firmly held by the lyophilic protein molecules. The concentration in unit volume is too great and the particles cannot be resolved by the ultramicroscope.

Weight and volume of the lens.-The weight of the human lens increases from an average weight of $0.175 \mathrm{gs}$. at the third decade to an average of 0.265 gs. in the eighth decade of life, an increase of over 50 per cent. Since the lens is a closed system, it must adapt itself to a more or less constant volume. This is only possible by a diminution in volume of the individual fibres. As the older fibres become more and more compressed by the steady addition of newly-formed fibres, there is a loss of fluid content and the fibres become increasingly sclerosed. This physiological hardening of the fibres cannot be compared to the formation of the horny layer of the epidermis. The chemistry of the two processes differs. The water content of the nuclear fibres of the lens and the soluble protein content is very much reduced in the ageing process.

\section{2.-Chemistry and Nutrition of the Lens}

Chemical constitution of lens.-The young adult lens consists of approximately 35 per cent. protein, 64.5 per cent. water, holding electrolytes and organic crystalloids in solution and 0.5 per cent. alcohol-ether soluble substances, sterols, lipides, fats and fatty acids.

Protein fractions of the lens.-The protein concentration of the lens is greater than that of any other body organ. There are two main fractions of the lens protein, a water insoluble fraction called albuminoid, and a water soluble fraction. The latter com- 
bines alpha-crystalline, beta-crystalline and gamma-crystalline. The gamma-crystalline, which has been isolated from the beta fraction, constitutes the total albumen content of the lens.

Protein changes in the normally ageing lens. $\rightarrow$ The relative concentrations of the two main fractions, as well as of the crystallines, change with age. For cattle lenses the ratio of soluble protein to insoluble protein changes from an initial $77: 23$ in the lenses of calves five weeks old to $41: 59$ in the lenses of 16 year old cattle.

Weight and water content of the lens.-The total weight of the lens increases with age. For cattle lenses the increase is from an initial weight of $0.91 \mathrm{gs}$. to a final weight of $2.7 \mathrm{gs}$. The total protein content increases from 32 per cent. ( 0.29 gs.) to 37 per cent. ( 1.0 gs.) There is therefore an absolute and a relative increase in protein content with age. Likewise, there is an absolute and relative increase in electrolytes and lipoids. The increase in water is only absolute; relatively the water content decreases with age. Thus the water content of cattle lenses decreases from a concentration of 76.4 per cent. ( 0.614 gs.) to 62.6 per cent. (1.9 gs.)

Lens protein fractions.-The shift in the ratio between soluble and insoluble protein fractions has been attributed to a transformation of the protein. This is rather difficult to reconcile with the established variations in concentrations of the amino-acids constituting the proteins of these groups. Whether the pressure, lack of nutritive and respiratory facilities to which the central fibres must be subjected, are sufficient to bring about such far reaching reconstruction or changes in the protein molecule, remains a matter of speculation. Loss in solubility does not necessarily demand a change in constitution, but may be brought about by the formation of stereo-isomers, that is, a change in the configuration of the protein molecule.

Isomers of the lens protein molecules.-The opportunities for isomeride formation of a protein molecule are almost unlimited. It has been postulated that the infinite variety of forms found in organic nature is partly the result of isomerism of the protein molecule. Assuming the presence of 15 asymmetric carbon atoms in any of the soluble protein of the crystallines, the possible stereoisomers of that protein are 1 or 32,768 in number. To this must be added the further possibility of tautomerism in the $-\mathrm{C}_{\mathrm{NH} 2}$ group of the amino-acids. This group may assume the tautomeric form $\mathrm{HO}-\mathrm{C}=\mathrm{N}$-and thereby give further opportunity for isomerism. A change in the structural formula may bring about loss in absorbing power for solvent, dehydration, exposure to enzyme action, change in state of aggregation, etc.

Hysteresis of the lens proteins.-The changes which the epithelial cells undergo in their gradual transformation into peripheral and later, nuclear fibres, will produce an hysteresis effect on the 
chief lyophilic constituent of the fibres, the proteins. The term hysteresis expresses the divergent behaviour of a colloid solution due to its previous treatment. The initial treatment of a protein solution may change certain of its physical properties, chiefly.its solvating ability and solubility. ${ }^{1}$.

Plasticity of lens protein.-Aside from the forces inherent in the progressive development of the lens, time itself has an effect on the viscosity and plasticity of lyophilic colloid solutions. The ageing of a colloidal system, such as a protein solution, seems to be accompanied by a decrease in its solvating properties and a consequent decrease in viscosity. The gradual transformation to a more stable isomeride producing the sclerosis of presbyopia may be due to the ageing of the protoplasmic proteins. It may be that a gradual fixation of a more stable form initiates age changes and prepares for the ultimate death and subsequent resolution of the cell to its constituent elements.

Other Chemical changes.-The effect of a gradually increasing electrolyte concentration, especially calcium, and the increase in concentration of the ether soluble constituents, on the physical state of the proteins must also be taken into account. To this must finally be added the cumulative effect of the products of lenticular metabolism, and the diminished rate of clearance of these products with increasing age and decreasing permeability of the lens and capsule.

Free and bound water.-Though the decrease in water content of the aged lens has been frequently demonstrated, there are so far no analyses of the free and bound water of the lens, and changes in the ratio of free to bound water which develop with age. A shift in the ratio in favour of free water may be assumed from (1) the known effect of age on the water binding capacity of protein, (2) the increase in calcium, fatty acids and sterols, (3) physical appearance of aged lenses.

Inorganic contents of the lens.-Coincident with the relative decrease in water content of the aged lens, the electrolyte concentration is increased. The ash of the lens increases from an initial concentration of 3 per cent. to approximately 3.8 per cent. This increase is mainly in the calcium and phosphorus. The calcium increases 15 per cent. of its initial value and the phosphorus 26 per cent. Part of the increase is derived from organic compounds of these ions.

The capsule has been reported as freely permeable to electrolytes. O'Brien and Salit ${ }^{2}$, report the chloride concentration of the ox-lens as averaging 356 mgs. per 100 gs. of wet material. The concentrations reported for the aqueous humour and vitreous by these authors were $709 \mathrm{mgs}$. and $681 \mathrm{mgs}$. per $100 \mathrm{gs}$. of material. This difference is considerable, even if the reduction in soluble space 
in the lens is taken into account. It would be interesting to determine the chloride content of calves lenses. In how far the demonstrated increase in cholesterol of aged lenses plays a part cannot be definitely stated. However, it is known that cholesterol decreases the ability of a tissue, other than the liver, to hold chloride.

Most important from the standpoint of permeability and solubility of the lens protein is the reported increase in calcium. Calcium crystals have frequently been found present in the nuclei of normal lenses, obtained post-mortem from patients above 50 years of age. Such crystals were not found in younger lenses. ${ }^{3}$.

Horning and Scott $t^{4}$, have shown that the distribution of inorganic constituents changes during the gradual development of the embryonic chick lens. "The calcium accumulates along the inner cavity of the lens, the iron salts appear to have migrated toward the periphery." This finding suggests the probable value of further work on the mineral skeleton of the lens at various ages, by the micro-incineration process of Policard ${ }^{5}$ and of Scott and Horning. ${ }^{6}$.

A shift in the relative concentrations of potassium and calcium in the growing eye has been demonstrated by Kaufman and Laskowski ${ }^{7}$. The calcium concentration increases and the potassium concentration decreases with age. The reduction in the $\mathrm{K} / \mathrm{Ca}$ ratio parallels the rate of growth. However, this phenomenon may also be an expression of the increasing sclerosis. If, analogous to the red blood corpuscles, calcium does not infiltrate the fibre, whereas potassium does, and is largely present in the interior only, then, as the ratio of volume to surface of the fibre decreases, the ratio of potassium to calcium must also decrease.

Organic constituents of the lens-glucose.-Among the organic metabolites, the glucose content of ox-lenses has been determined by O'Brien and Salit ${ }^{2}$ as 134.7 mgs. per 100 gs. of wet material. This is in excess of the glucose content of either aqueous humour (average $87 \mathrm{mgs}$.) or vitreous (average $56 \mathrm{mgs}$.) also determined by these authors. Part of the glucose content of the lens may have resulted from autolytic breakdown of cellular carbohydrate material. It may also be due to the presence of other reducing substances, such as glutathione in reduced form.

It has been calculated that the eye uses 438 mgs. glucose per day and that the oxygen available suffices for $200 \mathrm{mgs}$. only. The rest of the glucose is said to be broken down to lactic acid. The glucolytic breakdown of glucose within the lens is also indicated by the presence of lactic acid, in the aqueous humour in greater concentration than in the blood plasma. Furthermore, Kronfeld and Bothman ${ }^{8}$ in their experiments on the oxygen consumption of the surviving lens have shown that the lens, intra vitam, has an 
insufficient oxygen supply and has the property to glucolyse glucose under aerobic conditions. The surviving lens shows a minimal oxidative and quite marked glucolytic metabolism of carbohydrate material. Determination of glucolytic activity of sections of sterile cattle lenses by Michail and Vancea ${ }^{9}$ showed the activity of the cortical layer to exceed that of the sclerosed nucleus.

Lactic acid.-The lens in its capactity for lactic acid formation resembles a tissue cultured in vitro in which 40 per cent. of the total glucose consumed is converted to lactic acid only. The transient influence of the lactic acid on the lens protein should be recognized, even though the acid is promptly neutralized by lactate formation. The lactic acid radicle has a swelling influence on protein.

The auto-oxidation mechanism.-The lens possesses no circulatory system. It is surrounded by the intra-ocular fluids. For its nutrition as well as oxygen supply it depends entirely upon diffusion processes. Means for the conservation of the available oxygen supply are present in the auto-oxidation-reduction system of glutathione, thermo-stable reductor. It has been shown that glutathione is present in the lens and that it may be extracted with water or dilute acids, without hydrolysis of the proteins. Glutathione is a tri-peptide, glutamyl-cysteil-glycine. In the reduced form it contains a free sulphydryl group. Compounds containing this $-\mathrm{S}-\mathrm{H}$ group are important in tissue respiration and intracellular oxidation. The sulphur in this state readily undergoes partial oxidation to form the di-sulphide $(-\mathrm{S}-\mathrm{S})$ compound. In the presence of reducing substances this compound is again converted to the sulphydryl $(-\mathrm{S}-\mathrm{H})$, mono-sulphide state. In order that the glutathione may function as an auto-oxidation-reducing system, normal alkalinity of the lens protein and tissue fluid is essential.

Sulphur is a constituent of the soluble protein of the lens. The beta-crystalline fraction is richest in sulphur. However, in the protein molecule the sulphur is either present in the oxidized form or the sulphydryl group is protected from reacting since the lens from which the glutathione has been extracted no longer gives the reaction with sodium nitro-prusside, the qualitative test for the $\mathrm{S}-\mathrm{H}$ group.

The term "oxidation" is rather unfortunate as it seems to imply the actual participation of the element oxygen in the reaction and is therefore often confused with combustion. Chemically speaking oxidation ultimately means the assumption of a positive charge or the loss of a negative charge by an ion or atom. Every oxidation reaction involves a simultaneous reducing reaction. Viewing the oxidation of the sulphur in the cysteine radicle of glutathione from this standpoint will serve to clarify the relation of glutathione to 
the inner respiration of a tissue. Glutathione may be determined quantitatively by titrating its solution with a solution of molecular iodine. In this action the sulphur atom, which is negatively charged, is partly oxidized and the di-sulphide is formed. The iodine as the oxidizing agent is reduced to the ionic state and acts as the hydrogen acceptor. Likewise glutathione in the oxidized state may bring about oxidation of a compound by acting as hydrogen acceptor and being reduced to the sulphydryl state. The glutathione does not furnish oxygen for the combustion of sugar or other metabolites. It acts as a catalyst by forming the di-sulphide compond in the presence of molecular oxygen. In this state it acts as an hydrogen acceptor or in other words as oxidizing agent. In this respect it may be said to conserve oxygen, as it acts the part of the oxygen in the removal of the hydrogen. Since the oxygen supply is limited, the presence of such a compound as glutathione becomes important.

In the ageing lens the concentration of glutathione in the sulphydryl form is lessened. This would be in accordance with the decrease in oxidative processes within the lens substance, due to the increasing sclerosis of the lens fibres and decreasing vital activity.

The stimulating action of glutathione on cell proliferation has been demonstrated. Hammet and his co-workers have shown that the $-\mathrm{S}-\mathrm{H}$ group is the essential and natural stimulus to cell division. 10. The question thus presents itself as to how far a depletion of the sulphydryl compound of the lens effects the physiological maturing of the lens fibres.

Enzyme action in the lens.-Enzymes capable of accelerating oxidative processes are present in the lens as well as in the intraocular fluids. The presence of oxygenase, peroxidase as well as catalase has been demonstrated. These three enzymes represent a completely functioning oxidative system.

No systematic determination of the enzymes present in the lens has thus far been carried out. La Coscia found lipase, amylase, oxidase, catalase and glucolytic enzymes in the aqueous humour and in the vitreous. He also determined the presence of protease, catalase and lipase in the lens. ${ }^{17}$. Hayana determined the presence of proteases in the aqueous humour. ${ }^{18}$. The presence of these enzymes in the lens is likely. There may also be present phosphotases, which are said to be present in epithelial cells, as well as lecithinases, which are quite widely distributed.

Hydrogen-ion concentration of the lens.-The hydrogen-ion concentration, the $\mathrm{pH}$ of the lens has been determined potentiometrically by Scalini. ${ }^{11 .}$ The average $\mathrm{pH}$ of the lens was found to be 7.38. It lies, therefore, within the range of the $\mathrm{pH}$ variations for the aqueous humour, determined by Yudkin as 7:32 to 7.55.12. 
The normal $\mathrm{pH}$ range of venous blood plasma ranges from 7.33 to 7.55. Like other tissues, the normal lens maintains its $\mathrm{pH}$ within narrow limits.

The lipoids of the lens. - - The lipoids of the lens increase with age. The cholesterol content of the normal young-adult lens approximates that of the blood, i.e., 150 to $160 \mathrm{mgs}$. per $100 \mathrm{gs}$. of wet tissue. In old age this concentration may be more than doubled. Goldschmid extracted human lenses post-mortem with various organic solvents and studied the lipoids in the various fractions. The cholesterol was found to increase markedly from birth to the end of the second decade. This is followed by a slight decrease during the next three decades and a subsequent rise in the seventh decade. The phosphotide fraction decreased during the first two decades and then increased slightly, but never exceeded the concentrations found during the first few years of life..$^{13}$. There is a distinct shift in the relation of cholesterol to phosphotide in favour of cholesterol. Such a shift in ratio is of great importance from the point of view of cellular permeability and metabolism. G. Schlombke ${ }^{14,}$ found the cholesterol content of cattle lenses to increase from $196 \mathrm{mgs}$. per $100 \mathrm{gs}$. of wet material, during the first year of life, to $608 \mathrm{mgs}$. for aged cattle.

The sterols, cholesterol and its esters, are negatively charged, hydrophobic colloids. They are adsorbed on the cell surface, render the cell less permeable and isolate it electrically. According to Burger ${ }^{15}$, organs little or not at all supplied with capillaries are most easily impregnated with cholesterol. The lipides, on the other hand, are lyophilic colloids and have great swelling capacity. It has been shown that while cholesterol depresses cellular respiration, the phospholipin, lecithin, may act as oxygen carrier and have a stimulating effect on the inner respiration of a tissue. While the sterols in the environment of tissue cells and fluids have a physical function like all lipoids, the influence of sterols on cellular metabolism is much less marked than that of lipides. It seems that the fat metabolism of an organ is related to its phosphotide content and organs with a high fat metabolism also have high phosphotide content. Cholesterol, on the other hand, may retard fat metabolism. It inhibits lipase action probably by adsorbing the enzyme.

Among the vitamins of lipoidal nature vitamin $\mathrm{A}$ is present in the retina in relatively large concentration. Whether this vitamin is also present in the lens has not yet been determined.

Factors influencing the surfaces of the lens and its cells.-One may picture the cell surface as a mixture of proteins and lipoids.

* Lipoids are organic compounds, soluble in ether or other organic solvents. The group includes (1) Sterols, e.g., cholesterol. (2) Lipides, e.g., lecithin, sphingomyelin. (3) Cerebrosides, e.g., phrenosis. (4) Neutral fats, fatty acids and soaps. 
It is due to the proteins and hydrophilic lipoids present that the cell is permeable to water and dilute salt solutions. Owing to the colloidal nature of this protein-lipoid membrane, calcium exerts a permeability decreasing action. Potassium, by its peptizing influence, increases the permeability. The younger a cell, the less stable the formation of the surface layer and the more permeable the cell. Chambers showed that the membrane of a cell, as demonstrated by micro-dissection, has not a static, but a constantly varying structure. It can repair injury and the film, newlyformed, attains a certain rigidity through accumulations outside and inside of the cell. Calcium is necessary for the formation and maintenance of the membrane. ${ }^{16 .}$

With increase in cholesterol and calcium concentrations and the gradual breaking-up of the nucleus the lens cells become less and less permeable, the membranes assume a more and more unchanging character. The film thickens, the rate of exchange between interior and environment is decreased, until finally the isolated state of fibre is reached.

\section{3.-Analogous Changes in other Tissues}

The nutritive requirements of the tissue cell are supplied by the environment. Those cells which possess the best nutritive provisions will maintain themselves at the expense of the others, less well provided for. To the lack of nutritive material for the cells in certain parts of a given tissue must further be added the lack of adequate excretion and secretion. The same holds for the cell as such. The nucleus and the central part of a cell are the first to suffer from lack of nutrient material and insufficient removal of waste products. The resulting accumulation of catabolic material and cellular detritus initiates the ageing of a tissue. From time to time this excess, instead of being destroyed, is rejected in the form of a necrosis. The formation of such a "Nekron" is regarded by Kotsovsky as the beginning of age and death, as the death germ. ${ }^{19}$.

The structure of the lens renders it especially vulnerable to age changes. The lens relies upon diffusion processes, the surrounding ocular fluids supplying nutrient material and removing waste products. The conditions in the central part of the lens differ widely from those prevailing in the peripheral parts.

In the lens the fibres gradually lose their nuclei. The nuclei are first broken into fragments and these gradually disappear. Since the character of assimilation is determined by the nuclear apparatus, the aged lens, in which the ratio of nucleated fibres to non-nucleated fibres decreases, must show a gradual weakening 
of its metabolic processes. Waste pigment may also accumulate in the aged lens. The change in colour of the lens may be due partly to the greater consistency of the proteins and partly to waste pigment formation.

Chemistry of ageing.-The chemical processes of the physiological ageing of a tissue may be divided into:-(1) Primary changes; loss of water, thickening of substance, decrease in permeability, changes in diffusion. (2) Secondary changes; deposition of organic and inorganic waste material, accumulation of pigment, accumulation of sterols and fats.

Changes in bradytrophic tissues.-Burger and Schlombke ${ }^{20}$ chose bradytrophic tissue for the investigation of the chemistry of the ageing process. Bradytrophic tissue is that which has little or no supply of capillaries, such as certain layers of the aortic wall, cartilage, the sclera of the eye and in a certain sense, the ear drum. Such tissue shows significant age changes. Bradytrophic tissue in its uniformity of structure, resembles a non-living colloidal system more closely than other tissue. One may, therefore, expect to find certain analogies between the chemistry of the ageing process of such a tissue and the gradual transformation of a sol to a gel and the subsequent gradual loss in water binding capacity of the gel on ageing. Burger and Schlombke found the dry substance of human cartilage to increase from 24 per cent. in the first decade of life to 42 per cent. in the seventh decade. The changes in cholesterol concentration determined for human cartilage are analogous to the changes determined by Goldschmid for the lens. In both these tissues there is a rapid rise in cholesterol concentration during the first three decades of life, followed by a slight drop and a subsequent rise in old age. Both human cartilage and aorta show a close analogy in changes in water content, calcium and cholesterol to those of the ageing lens. ${ }^{21}$. Increasing dehydration was also shown for skin and sclera. The dry substance remaining after the evaporation of water changed in the sclera from 14.6 per cent. to 18.7 per cent.; in the skin from 28.6 per cent. to 35 per cent. of the original weight. In the lens the dry substance increases from 23.8 per cent. of the total weight to 37.4 per cent.

Loss of water, increase in calcium and cholesterol are primary changes in bradytrophic tissue, whether of ectodermal origin like the epidermis, lens or sclera, or of mesodermal origin like the aorta or cartilage.

The calcium and cholesterol concentrations of the blood are usually somewhat increased in old age. The cholesterol increases from an average of $142 \mathrm{mgs}$. per $100 \mathrm{ccm}$. of blood to an average of $182 \mathrm{mgs}$. Calcium also increases about 15 per cent. above its average value in early middle age. High cholesterol-ester 
content has often been found associated with tissue degeneration. The chemical disorganization of the lens fibres is often characterized by the appearance of anisotropic substances. (Jess ${ }^{22 .}$.)

There is a close parallelism between phosphotide content and mitochondria content of a tissue. Mitochondria plays an important part in the physiological activity of the cell, and cells physiologically active have a relatively high lipide content. (Cowdry 23 .) In the ageing lens the phosphotide content of the lens is decreased relative to the sterol and fatty acid content.

The signs of age in cells and tissues.-The signs of ageing tissue may be summarized as follows :-(1) Gradual dehydration, loss of water binding capacity, loss in permeability and changes in diffusibility. (2) Diminished metabolism. (3) Accumulation of waste material, incomplete recovery from fatigue. (4) Pigment formation. (5) Increase in sterol and calcium content. (6) A relative decrease in lipide content. (7) A possible decrease or loss in unsaturated fatty acid content. (8) A possible change in the configuration of the mineral skeleton of the cells.

The ageing process.-The ageing process is initiated as a local change and gradually spreads to the entire organism. Senile degenerative phenomena are homochronic. The separate organs and tissues follow in different forms a single law of ageing. Inheritance factors play a part in the difference in duration of life in individuals and species. In the same manner inheritance factors may play a part in the heterochronic ageing of an organ and the appearance of senility changes.

\section{4.-Discussion of Cataract}

Hypothesis of genetically determined cataract.-The genes are considered the carriers of inheritence factors. Their activity may be said to be due to certain specific areas, where the outwardly directed fields of force form an electric mosaic. The specificity of the gene depends not only on the chemistry of that particular area but also on the structural arrangement of the atom, molecule or ion in that portion. Any change in the solvent will register its effect not only on the configuration of the gene, but also on the molecular units coming within its jurisdiction. (J. Alexander 24.) The normal functioning of the genes therefore would demand the maintenance of the environment and an adequate supply and rate of removal of metabolic products.

Vogt maintains that the lens fibres are vitally differentiated and that their early or later degeneration is determined germ-plasmatically and inherited from generation to generation. ${ }^{25 .}$ The majority of the lens fibres have no nuclei; and vital differentiation may 
consist in a structural change of one or more of the protoplasmic components, imposed upon it by the genes, before destruction of the nucleus. The gene may have imparted a lasting effect on the electronic complexes, atoms, molecules and micelles in the environment. Pathogenic genes proteins may impart certain characteristics, which may render the lens fibre on absorption vulnerable to degenerative changes, dehydration, and the attack of proteolytic enzymes. If this hypothesis is tenable, then the tendency to senile cataract formation may be bestowed upon the lens fibres by the parent cells.

Structural changes in the lens in senile cataract.-For the purpose of this discussion, senile cataract may be divided into the hydrating type (senile cortical, posterior cortical, anterior and posterior subcapsular cataract) and into the sclerosing type (nuclear cataract).

Cortical cataract.-The early stages of the cortical types are characterized by a slight excess of fluid in the form of vacuoles, water-split sutures, separated lamellae, and ill-defined cortical haze. The fluid tends to follow certain lines of cleavage, both anterior and posterior, so that symmetrical areas are involved. Whether the fluid responsible for these phenomena is extruded from its combination with the lens protein or brought in through the capsule in excess of the normal amount is unknown. It is certain that in the later stages excess fluid is attracted through the capsule. The ill-defined haze in the cortex may be due to a cloudy swelling of the lens proteins within the fibres or to a lysis of the fibres with laking of the resultant fluid. In some instances it may simply be an oedema without any actual denaturation of the lens protein. The latter conception may explain the transient changes in the vision of some patients when the observer is unable to detect any change in the physical appearance of the vacuoles, split sutures or separated lamellae. Increase of the number of vacuoles, separated lamellae and split sutures marks the progress of cortical opacities. Greyish Morgagnian globules formed by the coagulation of the clear, colloidal fluid may develop. They are found in the split sutures and in the lakes of broken different stages of protein lysis, mixed with inorganic salts, sterols and fatty acids. At this stage the lens becomes swollen or intumescent. In the final stages the rapid death of the lens in shown by the autolysis of the fibres and proteins. Much of the material then goes into solution and is filtered off through the lens capsule. The lens which has been swollen then regains its normal size. The hypermature stages of the shrunken and Morgagnian types are produced by further digestion, extrusion and absorption of fluids and also by deposit of extraneous material. 
The concentration of lipoids is greatly increased in cataract. In the cataractous lens fatty degeneration probably follows abnormal fat infiltration, due to reduced oxidation processes. With the beginning of the degeneration of the fibres, this becomes true fatty degeneration. The double refractive bodies may also result from substances released by the degenerating fibres through enzymatic processes. The excess of cholesterol and fatty acids present would promote the formation of such double refracting bodies.

Physiological and pathological nuclear sclerosis (presbyopia and nuclear cataract).--In the physiological-nuclear sclerosis producing the familiar presbyopia, the process is smooth and there is no essential loss of transparency or change in the essential dioptric focus of the eye for distant vision. The colour of the lens by incident light is that of a very light grey, the lens being more relucent than in youth. In pathological-nuclear sclerosis, the process is uneven and lenticular myopia or second sight develops. A nuclear haze is seen by transmitted light, and a coloration develops which is amber in the early stages and goes through the reddish hues even to the formation of the brown or black cataract. The loss of nuclear transparency may be due to physical changesirregularities of the interfaces, pressure surfaces, or to chemical changes in the nuclear material. The clinical appearance of nuclear cataract is that of hardened, compressed fibres with diminished water content. The nature of the chemistry of the cataractous lens particularly of the nuclear type is similar to that of the normally ageing lens but greater in degree. The reduction in soluble protein content as well as the concentration of insoluble albuminoid is much increased. The cataractous lens loses its glutathione in whole or in great part. This parallels the decrease in inner respiration.

The numerous theories of the pathogenesis of senile cataract may be divided into four groups :-

(1) Functional, e.g., excessive accommodation, due to uncorrected errors of refraction.

(2) Biological, e.g., unequal sclerosis resulting in the separation of layers adjacent to the nucleus, decrease in regenerative processes of lens epithelium.

(3) Chemical, e.g., faulty nutrition due either to decreased permeability, metabolic disease or arterio-sclerosis, damage due to toxins or toxic catabolic products circulating in the blood, absorption of infra-red or ultra-violet rays, abnormal hormone or enzyme influences.

(4) Genetic, e.g., senile cataract being considered as the heterochronic appearance of one of the senile degenerative phenomena. 
The theory which regards senile cataract merely as an expression of early senility in the eye suffers from generality. Yet if one compares the chemistry and the structural changes of the normally ageing lens with those of certain types of cataract, one is impressed with the similarity of many changes, which qualitatively are alike and differ only quantitatively. Various factors such as starvation, insufficient oxygen supply, nervous influences, toxins or enzymes may initiate the changes which prepare cells for their dissolution. It is difficult to limit the aetiology of senile cataract formation to a single factor or to a group of factors. Changes in permeability of the capsule, of the lens fibre membranes, changes in diffusion in the fibres and in the interfibrillar spaces, changes in water binding properties of the lens protein, early loss of vital parts of the cells, abnormal infiltration with fat and with inorganic material may all play an important part in initiating a cataract.

The protoplasm of the lens fibres may bear the imprint of its genes in the structures of component molecules, atoms and micelles. The structural configuration of the proteins of the fibres derived from mother cells with pathologically altered genes may be such as to offer little, if any, resistance to proteolytic intra-cellular enzymes, or to the infiltration of lipoids. If, in addition, there is a local autochthonic factor for promoting the deposition of lipoids the stage is set for the beginning of the cataractous changes. If the constitution of the fibres is such as to confer upon it susceptibility to these changes which are able to produce opacity because of a weakening of the cellular or protoplasmic resistance, then cataract may be viewed as germ plasmatically determined.

Such a condition may be brought about either by hereditary weakness in the genes or by changes in the environment, which in turn weaken the genes. In this way one may explain the earlier occurrence of cataract in patients with hormonal and nutritional deficiency diseases or with diabetes mellitus. In this hypothesis concerning senile cataract formation, the primary change is a genetically induced change in the structural configuration of the protein molecule. The genealogy of senile cataract families may be difficult to work out due to variations produced by environment, but the hypothesis merits consideration.

\section{5.- Summary and Conclusions}

A study of the development, growth and structural changes, chemistry and nutrition of the normal lens in its relation to the aetiology of senile cataract is presented.

Analogous age changes in structure and chemistry of bradytrophic tissue are reviewed. 
The structural changes observed in senile cataract and the chemistry and nutrition of such lenses are discussed.

The theory which considers senile cataract germ-plasmatically determined is elaborated upon. Pathological changes in the structural configuration of the lens protein molecules predispose to a lack of resistance of the lens as an organ, to changes in permeability, to changes in water binding power and tofatty degenerations. These have an effect of promoting degenerative phenomena of denaturation, coagulation, and precipitation of lens protein and of inducing abnormal infiltration of fat and deposition of inorganic material. This hypothesis considers senile cataract to be a genetically induced change in the structural configuration of the lens protein molecule which makes it susceptible to the physical and chemical phenomena concerned in the production of cataract.

\section{BIBLIOGRAPHY}

1. Robertson, T. B.-Physical Chemistry of the proteins. Longmans, Green and Co., N.Y. 1918.

2. O'Brien, C. S., and Salit, P. W.-Amer. Jl. of Ophthal., Vol. XIV, p. 582, 1931.

3. Goldschmid, M.-Klin. Monatsbl. f. Augenheilk., Vol. LXXII, p. 777, 1924.

4. Horning and Scott.-Anat. Rec., Vol. LII, p. 351, 1932.

5. Policard.-Anat. Rec., Vol. XLIV, p. 349, 1930.

6. Scott and Horning.-Amer. Jl. of Pathol., Vol. VIII, p. 329, 1932.

7. Kaufman and Laskowski.-Biochem. Zeitschr., Vol. CCXLII, p. 424, 1931.

8. Kronfeld, P., and Bothman, L.-Zeitschr. f. Augenheilk., Vol. LXV, p. 41, 1928.

9. Michail, D., and Vancea, P.-Compt. Rend. Soc. Biol., Vol. CIX, p. 1011, 1932.

10. Hammond, F. S.-Protoplasma, Vol. XIII, p. 331, 1931.

11. Scalinci.-Boll. Soc. Biol. sper., Vol. IV, p. 359, 1929.

12. Yudkin, A. M.-Arch. of Ophthal., Vol. I, p. 615, 1929.

13. Goldschmid, M.-Biochem. Zeitschr., Vol. CXXVII, p. 210, 1922.

14. Schlombke, G.-Med. Klin., Vol. XXVI, p. 1065, 1930.

15. Buerger, W., and Schlombke, G.-Zeitschr. f. d. ges. $\exp$.Med., Vol. LV, p. $286,1930$.

16. Chambers, R.-Ann. Physiol. physico-chem-biol., Vol. VI, p. 233, 1930.

17. La Coscia, G. L.-Ann. di ottal. e clin. oculist, Vol. L, p. 219, 1922:

18. Hayana-Quoted in Die Fermente und ihre Wirkungen, C. Oppenheimer. Georg Thieme, Leipzig, 1924.

19. Kotsovsky, D.-Ergebn. d. Physiol., Vol. XXXI, p. 133, 1931.

20. Buerger, W., and Schlombke, G.-Op. cit.

21. Schlombke, G. -Op. cit.

22. Jess, A.-Kurzes Handb. d. Ophthal., Vol. V. J. Springer, Berlin, 1930,

23. Cowdry, E. V.-Quoted by D. J. Koogan, Arch. of Dermatol., Vol. XXV, p. 235,1932 .

24. Alexander, Jerome.-Protoplasma, Vol. XIV, p. 286, 1931.

25. Vogt, A.-Lehrbuch und Atlas der Spaltlampenmikroscopie. J. Springer, Berlin, 1928. 